\title{
Using a sensitivity study to facilitate the design of a multi-electrode array to measure six cardiac conductivity values
}

\author{
Barbara M. Johnston \\ School of Biomolecular and Physical Science and Queensland Micro and Nanotechnology \\ Centre \\ Griffith University \\ Nathan \\ Queensland \\ Australia, 4111 \\ Phone: $61-7-3735-4405$ \\ Fax: 61-7-3735-7656
}

\begin{abstract}
When using the bidomain model to model the electrical activity of the heart, there are potentially six cardiac conductivity values involved: conductivity values in directions along and normal to the cardiac fibres with a sheet, as well as a conductivity value in the normal direction between the sheets, and these occur for both the extracellular and intracellular domains in the model. To date it has been common to assume that the two normal direction conductivity values are the same. However, recent work has demonstrated that six cardiac conductivity values, rather than four, are necessary for accurate modelling, which can then facilitate understanding of cardiovascular disease.

To design a method to determine these conductivities, it is also necessary to design a suitable multi-electrode array, which can be used, in conjunction
\end{abstract}

Email address: Barbara.Johnston@griffith.edu.au (Barbara M. Johnston) 
with an inversion technique, to retrieve conductivity values from measurements of potential made on the array. This work uses the results of a study, into the sensitivity of the measuring potentials to variability in the input conductivities, to facilitate the design of an array that could be used to retrieve six cardiac conductivity values, as well as fibre rotation angle.

It is found that if an electrode in the array has a much lower value of potential than the other electrodes, then it tends to be much more sensitive to the input conductivities than the other electrodes. It also appears that inclusion of this type of electrode in the set of measuring electrodes is essential for accurately retrieving conductivity values. This technique is used to identify electrodes to be included in the array and using the final design it is demonstrated, using synthetic values of potential, that the six cardiac conductivity values, and the fibre rotation angle, can be retrieved very accurately.

Keywords: bidomain model, conductivity values, sensitivity study, electrode array, simulation

\section{Introduction}

The bidomain model [1] is used extensively in computational cardiac physiology to study electrical activity in the heart. It considers the cardiac tissue as consisting of two interpenetrating regions, whose properties are averaged in each of the intracellular and extracellular domains. Until relatively recently, these models have generally made the simplifying assumption that cardiac conductivity, within a particular domain, is the same in the two directions that are normal to the cardiac fibres, one within the fibre sheet and 
the other between the sheets of fibres $[2,3,4,5]$. Since there is a third direction in which current can flow (along the fibres) this leads to four cardiac conductivity values rather than the full six values.

However, recent work has found [6,7] that this assumption should not be made and that it is necessary to use six values. For example, when studying partial thickness ischaemia, the epicardial distributions, modelled using six independent cardiac conductivity values, are a much better match for experimental data than those modelled under the assumption of equal normal and transverse conductivities [8].

Few sets of experimentally determined cardiac conductivity values are available $[9,10,11]$ and these vary quite widely in value [12] and produce very different distributions when used in modelling studies [13, 14]. These are all sets of four conductivity values and the sets of six conductivity values that are mentioned in earlier work $[15,7]$ are not determined experimentally.

Previously the author and others $[16,17,18]$ have demonstrated, using simulated data, that a two-pass inversion technique, combined with measurements made on a two-dimensional (2D) multi-electrode array, can be used to accurately retrieve four conductivity values, as well as the fibre rotation angle through the tissue. This study presents an extension of that work to a three-dimensional (3D) array in Sections 2-3, so that both the fibre rotation angle and six conductivity values can be retrieved. The focus of this paper is the design of the $3 \mathrm{D}$ array and to that end a sensitivity study is carried out to examine the effect of variability, in the input conductivities, on the potentials measured on the array (Section 4). This leads to the proposal of a method for deciding which measuring electrodes should be included in the 
modified versions of the array that are then considered. A summary of the results is given in the final section along with suggestions for future work.

\section{Model}

\subsection{Governing Equations}

The model used here represents cardiac ventricular tissue as a $2 \mathrm{~cm} \times 2$ $\mathrm{cm} \times 1 \mathrm{~cm}$ block in the $x, y$ and $z$ coordinate directions, respectively (Figure 1). The outer surface of the heart (epicardium) is the $x-y$ plane and the $z$ coordinate axis is positive downwards, with the inner surface of the heart (endocardium) represented by the plane at $z=1$. At that point, the endocardium meets an infinite volume of blood, which extends in the positive $z$ direction (Figure 1).

A passive bidomain model is used to approximate the cardiac tissue, during the ST segment, where it is assumed that the electric potentials do not change with time. This gives the following [1] governing equations:

$$
\nabla \cdot\left(\mathbf{M}_{i} \nabla \phi_{i}\right)=\frac{\beta}{R}\left(\phi_{i}-\phi_{e}\right) \quad \text { and } \quad \nabla \cdot\left(\mathbf{M}_{e} \nabla \phi_{e}\right)=-\frac{\beta}{R}\left(\phi_{i}-\phi_{e}\right)-I_{s}
$$

where $\phi_{e}$ is the extracellular potential, $\phi_{i}$ is the intracellular potential, $\beta$ is the surface to volume ratio of the cells, $R$ is the specific membrane resistance and $I_{s}$ is the external current source per unit volume applied in the extracellular space. The tensors $\mathbf{M}_{e}$ and $\mathbf{M}_{i}$ reflect the anisotropic conductivity, as well as fibre rotation, within the cardiac tissue [13], for the extracellular and intracellular domains, respectively.

Also, Laplace's equation governs the potential distribution in the blood, 
$\phi_{b}$,

$$
\nabla^{2} \phi_{b}=0
$$

\subsection{Conductivity Tensor}

Cardiac tissue consists of sheets of parallel fibres (strands of cells) and is electrically anisotropic, since current flows more easily, within the sheet, along the fibres (longitudinal $=l$ ) compared with across the fibres (transverse $=t$ ). Current is also able to flow between the sheets $($ normal $=n$ ). In addition, the bidomain model [1] assumes that the tissue can be treated as two contiguous domains, intracellular $(i)$ and extracellular $(e)$. This leads to six bidomain electrical conductivities: $g_{i l}, g_{i t}, g_{i n}, g_{e l}, g_{e t}$ and $g_{e n}$.

It is common [19] to assume that the cardiac fibres rotate linearly with depth between the epicardium and the endocardium, which leads to the following form for the conductivity tensors

$$
\mathbf{M}_{p}(x, y, z)=\left(\begin{array}{ccc}
\left(g_{p l}-g_{p t}\right) c^{2}+g_{p t} & \left(g_{p l}-g_{p t}\right) c s & 0 \\
\left(g_{p l}-g_{p t}\right) c s & \left(g_{p l}-g_{p t}\right) s^{2}+g_{p t} & 0 \\
0 & 0 & g_{p n}
\end{array}\right)
$$

where the fibre rotation angle is taken to be $\alpha, s=\sin \alpha z, c=\cos \alpha z$ and $p=i$ or $e$.

\subsection{Boundary Conditions}

The following boundary conditions are used to solve the model. Firstly, assume that the epicardium is insulated, giving

$$
\text { at } z=0 ; \frac{\partial \phi_{e}}{\partial z}=\frac{\partial \phi_{i}}{\partial z}=0 .
$$


Next, assume that there is continuity of potential and current at the interface between the tissue and the blood, and that the intracellular space is insulated by the extracellular space, giving

$$
\text { at } z=1 ; \phi_{e}=\phi_{b}, \quad g_{b} \frac{\partial \phi_{b}}{\partial z}=g_{e n} \frac{\partial \phi_{e}}{\partial z}, \quad \frac{\partial \phi_{i}}{\partial z}=0
$$

where $g_{b}$ is the conductivity of blood and $\phi_{b}$ is the potential in the blood. Also, $\phi_{b} \rightarrow 0$ as $z \rightarrow \infty$ since the blood mass is assumed infinite in the positive $z$ direction. Finally, assume that the boundaries of the domain are insulated, giving at the $x$ and $y$ boundaries

$$
\mathbf{M}_{e} \nabla \phi_{e} \cdot \mathbf{n}=0, \mathbf{M}_{i} \nabla \phi_{i} \cdot \mathbf{n}=0 \text { and } \nabla \phi_{b} \cdot \mathbf{n}=0
$$

where $\mathbf{n}$ is the normal which points outwards from the boundary.

\subsection{Solution Technique}

The solution technique for the forward model is very similar to previous work $[16,18]$ and involves expansion of $\phi_{i}$ and $\phi_{e}$ as a Fourier series. Then a one-dimensional finite difference scheme is used to find the series coefficients [16]. An advantage of this solution technique is that the potential at any

point in the domain (for example, an electrode) can be found as the sum the series there.

\subsection{Modelling Parameters}

Parameters used here are $\beta=2000 \mathrm{~cm}^{-1}, R=9100 \Omega \mathrm{cm}^{2}, I_{S}=50 \mu \mathrm{A} / \mathrm{mm}^{3}$, $\alpha=2 \pi / 3$ and $g_{b}=6.7 \mathrm{mS} / \mathrm{cm}$, consistent with previous work [18]. Two sets of conductivity values are used in this study: the set of MacLachlan et al. 
[15], which is derived from the work of Foster and Schwan [20], and the set of Hooks et al. [7] (Table 1). This results in space constants $\lambda_{q}(q=l, t, n)$, where $\lambda_{q}=\sqrt{\frac{R}{\beta}\left(\frac{g_{i q} g_{e q}}{g_{i q}+g_{e q}}\right)}$, which lie between $0.18 \mathrm{~mm}$ and $0.77 \mathrm{~mm}$ (Table 1 ).

\section{Methods}

\subsection{Inversion Process}

Since the measured potentials $\boldsymbol{\Phi}$ depend non-linearly on the parameters $\mathbf{m}=\left[g_{i l}, g_{i t}, g_{i n}, g_{e l}, g_{e t}, g_{e n}, \alpha\right]^{T}$ (equation 1), and there will be noise in the measured potentials, the inversion technique for the first pass is based on minimising the Tikhonov functional

$$
\|\mathbf{G}(\mathbf{m})-\boldsymbol{\Phi}\|_{2}^{2}+\gamma_{1}^{2}\left[g_{i l}^{2}+g_{i t}^{2}+g_{\text {in }}^{2}+g_{\text {el }}^{2}+g_{\text {et }}^{2}+g_{\text {en }}^{2}\right]+\gamma_{2}^{2} \alpha^{2}
$$

where $\mathbf{G}$ represents the forward model and $\gamma_{1}$ and $\gamma_{2}$ are the regularisation parameters. The differences between $\mathbf{G}(\mathbf{m})$ and $\boldsymbol{\Phi}$ are calculated at a set of the electrodes on the measuring array. In a similar fashion the Tikhonov functional

$$
\|\mathbf{G}(\mathbf{m})-\boldsymbol{\Phi}\|_{2}^{2}+\gamma_{1}^{2}\left[g_{i l}^{2}+g_{i t}^{2}+g_{i n}^{2}\right]+\gamma_{2}^{2} \alpha^{2}
$$

is minimised in the second pass.

The functionals are minimised using the SolvOpt solver [21], which uses a modified version of Shor's $r$-algorithm. Shor's minimisation method with space dilation efficiently minimises non-linear multivariate functions using the method of exact penalisation to handle constraints [22]. The modifications to this method include the addition of an efficient stopping criterion and a method for choosing the initial step size [21]. 
In previous work [18], the regularisation parameters were taken to be $\gamma_{1}=10^{-2}$ and $\gamma_{2}=10^{-5}$, partly based on the relative magnitudes of the conductivities compared with the fibre rotation angle and after testing of other values. These same values were tested initially in this study, because of the similarity of the functional to that in the previous study and, since the method was found to be relatively insensitive to the the choice of regularisation parameter, the same values were used in this study.

\subsection{D Array and Method for Retrieving Four Conductivities}

The electrode array proposed in this work is an extension of an array already published by the author and others [18], which was designed to retrieve the four conductivities $\mathrm{g}_{i l}, \mathrm{~g}_{i t}, \mathrm{~g}_{e l}$ and $\mathrm{g}_{e t}$ from measurements of potential, as well as fibre rotation $\alpha$. That array consisted of 17 micro-needles [18], each containing two electrodes, lying in two planes. The dimensions of the array were chosen so that potential measurements could be made at distances less than or equal to the space constant of the tissue ('closely spaced'), as well as at distances greater than the space constant ('widely spaced') (Table 1), consistent with previous work [2] that showed that this is necessary to find both types of conductivities.

Our work with the 2D array found that it is necessary to use a two-pass protocol to accurately retrieve the desired parameters [18]. During the first

pass, to find the extracellular conductivities, potentials are stimulated and measured on a subset of 'closely spaced' $(0.5 \mathrm{~mm})$ electrodes [2]. The second pass then uses 'widely spaced' electrodes $(1 \mathrm{~mm})$ to find the intracellular potentials, with extracellular potentials from the first pass held constant. 


\subsection{D Array and Method for Retrieving Six Conductivities}

\subsubsection{Electrode Array}

The proposed design (Figure 2), for an array to retrieve six conductivity values, is a three dimensional analogue of the array described in Section 3.2. The 'closely-spaced' electrode set (25 measuring electrodes plus source and sink electrodes) consists of three layers of electrodes, spaced $0.5 \mathrm{~mm}$ apart in all directions (Figure 3(a)). Two 'widely-spaced' electrode sets are also considered, again with spacing in the normal direction of $0.5 \mathrm{~mm}$, the first with 37 measuring electrodes (Figure 3(b)) and the second with 73 measuring electrodes (Figure 3(c)).

\subsubsection{First Pass}

The parameters from Section 2.5, together with the conductivities of MacLachlan et al. [15] and the 'closely-spaced' electrode set from Figure 3(a), are used to produce a set of simulated potential measurements. Next three lots of ten sets of 'noisy' potentials are produced by adding $1 \%, 2 \%$ and $5 \%$ noise to the original potentials. The inversion technique described in Section 3.1 is then used to retrieve all seven parameters $\left(g_{e l}, g_{e t}, g_{e n}, g_{i l}, g_{i t}, g_{i n}\right.$ and $\alpha$ ) simultaneously during the first pass through the solver. Initial values are $\alpha=0.5$ and all the $g_{e}$ and $g_{i}$ values are $1 \times 10^{-3}$, except for $g_{i n}=1 \times 10^{-4}$.

\subsubsection{Second Pass}

A similar set of simulated potentials is calculated for 'widely-spaced' grid 1 (Figure 3(b)) and then noise is added in a similar fashion to above before the inversion is carried out. However, in this case, the mean values for $g_{e l}, g_{e t}$ and $g_{e n}$ from the first pass are held constant in the inversion and only values 
for $g_{i l}, g_{i t}, g_{i n}$ and $\alpha$ are retrieved. Their starting values in this case are their means from the first pass. Once again a set of 10 inversions is completed for each noise level and the means from this set are the final values for the intracellular conductivities and the fibre rotation angle.

\subsection{Limitations of the Model and Inversion Process}

The limitations and assumptions of the model and the methods described above include such issues as modelling fibre rotation, the assumption of an imbrication angle of zero, and the alignment of the measuring array. These issues are similar to those in the previous work [16, 18] and a full discussion can be found therein. The latter study [18] also includes a discussion of the practical difficulties associated with manufacturing and calibrating arrays with such small electrode spacings and describes techniques that have been developed to deal with this.

\subsection{Sensitivity Analysis}

An error of $10 \%$, chosen to be of the same order of magnitude as the noise that is added to the conductivities in the simulations in Section 3.3, is added to a particular conductivity value before calculating the potential on the set of electrodes. Then the percentage relative error in $\phi_{e}$ (sensitivity), for that conductivity value, is calculated at each electrode, where

$$
\text { sensitivity }=\left|\frac{\phi_{e}-\phi_{e c}}{\phi_{e}}\right| \times 100 \%
$$

and $\phi_{e}$ is the simulated potential at a particular electrode and $\phi_{e c}$ is the simulated potential at the same electrode, once the $10 \%$ error has been added to the conductivity value. 


\section{Results and Discussion}

\subsection{Electrode Sensitivity Analysis}

The sensitivity of each of the electrodes, in both the 'closely-spaced' and the first 'widely-spaced' set of electrodes in the proposed array, is investigated for the data of MacLachlan et al. [15] (Figure 3(a) and (b)). This is achieved by adding $10 \%$ error to each of the six conductivity values individually. Examples of this are shown in Figure 4 for (a) the 25 'closely-spaced' measuring electrodes shown in Figure 3(a) and (b) the 37 'widely-spaced' measuring electrodes in Figure 3(b). (Sensitivity is not plotted for the final electrode in each array as that electrode is always taken to be the reference potential).

Most electrodes have sensitivity values of $15 \%$ or less to $10 \%$ error added to any of the conductivity values, with the sensitivities to intracellular error generally around $5 \%$ or less. In Figure 3(a), the exceptions are electrode \#2, which shows high sensitivity to error in all three extracellular conductivities, as well as electrodes \#8, 16 and 19 which are sensitive to both $g_{e l}$ and $g_{\text {et }}$. Sensitivity values are between $30 \%$ and $70 \%$ on electrode $\# 2$ for the extracellular conductivities. For 'widely-spaced' electrode set 1 in Figure $3(\mathrm{~b})$, the exception is electrode \#3, which is particularly sensitive to error in the extracellular conductivities, with sensitivity values between $g_{e t}=130 \%$ and $g_{e l}=280 \%$. In addition, this electrode is also quite sensitive to error in the intracellular conductivities, with values between $25 \%$ and $55 \%$, in

comparison to the other electrodes, where the intracellular sensitivity values are all less than $6 \%$.

Previous work [2] has shown that almost all the current is found in the 
extracellular space, when using 'closely-spaced' measuring electrodes, and in both domains when using 'widely-spaced' electrodes. The work here is consistent with this, in that the most sensitive electrode in the 'closelyspaced' case is sensitive to error in the extracellular conductivities (only), whereas the most sensitive electrode in the 'widely-spaced' case is sensitive to both types of conductivities.

The previous array [18], that was used to successfully retrieve the four conductivity values (Section 3.2), was initially used to try to retrieve six conductivities (and fibre rotation) and was found to work very well in the first pass to find the extracellular conductivities, including $g_{e n}$, but it did not work well in the second pass. That array was also analysed in a similar fashion to the above and very high sensitivity values (over 1000\%), to the extracellular conductivities, were found for a particular electrode in the 'closely-spaced' set, consistent with the above results. It is not surprising that the array did not work well in the second pass since it has only two electrodes on each needle, that is, in the normal direction.

Other sensitivity experiments were performed to investigate adding error to more than one conductivity value at a time. There was a near-linear response to this on almost all the electrodes, except the very sensitive electrodes where the response was magnified, underlining the importance of the very sensitive electrodes and indicating that the conclusions drawn here would not be affected by continuing with the method of adding error individually to the conductivity values.

An alternative way of analysing the electrode sensitivity is to plot sensitivity against $\phi_{e}$ for all the electrodes and conductivities. Such plots (on 
a $\log$ scale) are shown in Figure 5 for (a) the 'closely-spaced' electrode set and (b) 'widely-spaced' electrode set 1 (Figure 3). These plots correspond to those in Figure 4, although they do not indicate which particular electrode is the most sensitive.

Study of these and other similar plots (for example, for the dataset of Hooks et al. [7], which are not presented) leads to the following conclusions:

1. Electrodes are more sensitive to error in the extracellular conductivity values than to error in the intracellular values;

2. Different electrodes are the most sensitive when considering 'closelyspaced' or 'widely-spaced' electrode sets;

3. The 'closely-spaced' set of electrodes is sensitive to error in the extracellular conductivity values, but not to intracellular values, whereas the most sensitive electrode in the 'widely-spaced' electrode set is sensitive to both extracellular and intracellular conductivity values.

4. The same electrode in the 'widely-spaced' set is the most sensitive for both the intracellular and extracellular conductivities.

5. The most sensitive electrode is generally the one where the potential $\phi_{e}$ is lowest.

An investigation was also carried out where the most sensitive electrode was removed from a set and the inversion process to retrieve the conductivities was repeated. It was found that the process with the sensitive electrode excluded was much less accurate than using the set that included that electrode. This information was then used to choose electrodes to be included in some new designs for the electrode arrays. 


\subsection{New array design for the 'closely-spaced' electrode set}

The first modification simply involves reducing the electrode spacing, from $0.5 \mathrm{~mm}$ to $0.25 \mathrm{~mm}$, in the normal direction only, in the 'closely-spaced' electrode set. The idea is suggested by the smaller values for $\lambda_{n}$ than for the space constants in the other two directions (Table 1). A plot of sensitivity versus $\phi_{e}$, for the new electrode set and the data of MacLachlan et al. [15], is shown in Figure 6. Interestingly, the most sensitive electrode moves from \#2 for the $0.5 \mathrm{~mm}$ spacing to \#1 for the $0.25 \mathrm{~mm}$ spacing (Figure 3(a)). A comparison of this plot with the one using the original $0.5 \mathrm{~mm}$ spacing in Figure 5(a) shows a large improvement in mean sensitivity, on the most sensitive electrode for the three extracellular conductivities, from around 50\% to $1500 \%$.

The first pass inversion process is carried out in full, for the MacLachlan et al. [15] dataset, for this new design (Table 2). Note that this is a first pass and so the poor values for the intracellular conductivity errors are to be expected. The extracellular conductivities, for the new $0.25 \mathrm{~mm}$ spacing, are retrieved with much the same, or even slightly better accuracy than in the $0.5 \mathrm{~mm}$ case, and so are the $g_{i l}, g_{i t}$ (except for $5 \%$ noise) and $\alpha$ values. However, the $5 \% g_{i t}$ and all the $g_{i n}$ values are much more inaccurate, causing a problem because these values are needed to provide the initial values for the second pass.

\subsection{New array design for the 'widely-spaced' electrode set}

This modification involves including all 73 electrodes in the array for the second pass (Figure 3(c)), since the conductivity values are not known $a$ priori and so the electrode with the lowest potential cannot be predicted. In 
fact, the electrode with the lowest value of $\phi_{e}$ is different for the conductivity data of MacLachlan et al. [15] (\#3 in Figure 3(b)) compared with Hooks et al. [7] (\#17 in the same figure). Using 73 rather than 37 electrodes has a negligible effect on the computing time, due to the solution method.

When sensitivity plots for 37 electrodes (Figure 7(a)) are compared with those for 73 electrodes (Figure 7(b)), for the data of Hooks et al. [7], it is found that there are, in fact, three electrodes that are more sensitive than the most sensitive one in the 37 electrode set. The most sensitive electrode in the new set is now \#14 (Figure 3(c)), which is not part of the 37 electrode set (Figure 3(b)). This new set results in an improvement in the mean extracellular sensitivity on the most sensitive electrode from approximately 100 to 2000, while the mean intracellular sensitivity increases from approximately 10 to 200. This is not the case, however, for the MacLachlan et al. data, as the most sensitive electrode (\#9 in Figure 3(c)) is already in the electrode set (\#3 in Figure 3(b)).

The second pass inversion process is now repeated using an array containing 73 electrodes, for both the data of MacLachlan et al. [15] and that of Hooks et al. [7] (Table 3). It is found that the conductivities and fibre orientation angle cannot be retrieved quite as accurately in every case, using 73 rather than 37 electrodes, for the MacLachlan et al. [15] data, whereas, for the Hooks et al. [7] data, using 73 electrodes produces better results. The former result is not really a problem as the results are still quite accurate, while the latter result is not unexpected as the 73 electrode set now includes the most sensitive electrode for the case of Hooks et al. [7], unlike MacLachlan et al. [15] where the electrode was already included. 


\section{Conclusions}

This work discusses the design of a multi-electrode array to be used, in conjunction with a bidomain model and an inversion technique, to retrieve six cardiac conductivity values from measurements of potential on the heart surface. Sensitivity, to error added to the conductivity values, is studied for 'closely-spaced' and 'widely-spaced' electrode sets in the proposed array. In each case, one or more particularly sensitive electrodes are found and it seems that the presence of at least one of these electrodes is crucial for the conductivities to be retrieved accurately. However, it is found that the position of the most sensitive electrode is not usually the same in the first and second pass subsets of electrodes, or when different generating conductivity datasets are used.

Analysis, of the relationship between sensitivity and potential measured on an electrode, has found that the most sensitive electrode is almost always the one with the lowest potential. This relationship, between sensitivity and potential on an electrode, then provides a technique for analysing various different arrangements of electrodes, and, based on this technique, two modifications to the array are proposed.

The first involves decreasing the electrode spacing from $0.5 \mathrm{~mm}$ to 0.25 $\mathrm{mm}$, in the normal direction only, for the 'closely-spaced' electrode set. However, when the first pass of the inversion process is carried out, it is found that, although the extracellular conductivities are retrieved more accurately by the new array, it is ineffective at high noise levels, for the intracellular

conductivities, and is particularly poor at retrieving $g_{i n}$. It would appear that there needs to be a trade-off between the accuracy of the extracellular 
conductivities and the intracellular conductivities retrieved in the first pass. In view of the need to ensure that there are sufficiently accurate starting values for the intracellular conductivities for the second pass, this modification is not recommended.

The second modification suggested is to use all 73 available electrodes in the second pass to retrieve the intracellular conductivities to allow for the fact that it is not possible to predict which electrode will be the most sensitive ahead of time. This is not an issue in the first pass because a full set of 'closely-spaced' electrodes is already being used. This 73 electrode approach was found to be satisfactory by repeating the second pass of the inversion process and it is recommended that it be incorporated into the design of the multi-electrode array.

Further work in this area could include the use of the technique described above to study aspects of the array design, such as source and sink placement, the number of electrodes and spacing between the electrodes.

\section{Acknowledgements}

This work was supported in part by a Griffith University New Researcher Grant. 


\section{Captions}

Figure 1: Two views of the tissue-blood model used in this work. In the cross-sectional view in the left panel, the positive $z$ axis is pointing downward, with the tissue lying between $z=0$ and $z=1$ and the blood at $z>1$. In the right panel is a plan view, which looks from the negative $z$-direction, the dashed arrow represents the endocardial $(z=1)$ fibre direction, when the epicardial $(z=0)$ fibre direction is along the $x$-axis and the fibre rotation angle is $\alpha$.

Figure 2: The full three-layer multi-electrode array proposed for retrieving six conductivity values. A different subset of the electrodes is used in each of the passes in the inversion (see Figure 3).

Figure 3: Plan view of the three layers of the multi-electrode array, shown in Figure 2, that are used in (a) the first pass and (b) and (c) in alternative electrode sets in the second pass of the protocol to retrieve the six conductivities. The spacing between the marked squares is $0.5 \mathrm{~mm}$ in each case. Source and sink electrodes are indicated by + and - respectively.

Figure 4: Sensitivity (Percentage relative error in $\phi_{e}$ ), when $10 \%$ error is added to the conductivity values, for (a) the 'closely-spaced' and (b) 'widelyspaced' electrode set 1 of Figure 3, for the MacLachlan et al. [15] conductivities.

Figure 5: Sensitivity plotted against $\phi_{e}$, when $10 \%$ error is added to the conductivity values, for the MacLachlan et al. [15] conductivities. The 'closelyspaced' electrode array is the same as that used in Figure 3(a), except that the electrode spacing in the normal direction is now $0.25 \mathrm{~mm}$.

Figure 6: Sensitivity plotted against $\phi_{e}$, when $10 \%$ error is added to the 
conductivity values, for the Hooks et al. [7] conductivities. The 37 electrode array ('widely-spaced' electrode set 1) is shown in Figure 3(b) and the 73 electrode array ('widely-spaced' electrode set 2) is shown in Figure 3(c). 


\section{References}

[1] O. H. Schmitt, Biological information processing using the concept of interpenetrating domains, in: K. N. Leibovic (Ed.), Information Processing in the Nervous System, Springer-Verlag, New York, 1969, pp. $325-331$.

[2] R. Plonsey, R. C. Barr, The four-electrode resistivity technique as applied to cardiac muscle, IEEE Trans. Biomed. Eng. 29 (1982) 541-546.

[3] R. Sadleir, C. Henriquez, Estimation of cardiac bidomain parameters from extracellular measurement: Two dimensional study, Annals Biomed. Eng. 34 (2006) 1289-1303.

[4] A. E. Pollard, R. C. Barr, Cardiac micro-impedance measurement in two-dimensional models using multisite interstitial stimulation, Am. J. Physiol. Heart Circ. Physiol. 290 (2006) H1976-H1987.

[5] A. E. Pollard, C. D. Ellis, W. M. Smith, Linear electrode arrays for stimulation and recording within cardiac tissue space constants, IEEE Trans. Biomed. Eng. 55 (2008) 1408-1414.

[6] D. A. Hooks, K. A. Tomlinson, S. G. Marsden, I. J. LeGrice, B. H. Smaill, A. J. Pullan, P. J. Hunter, Cardiac microstructure: Implications for electrical propagation and defibrillation in the heart, Circ. Res. 91 (2002) 331-338.

[7] D. Hooks, Myocardial segment-specific model generation for simulating the electrical action of the heart, BioMed. Eng. OnLine 6 (2007) 21-21. 
[8] P. R. Johnston, Cardiac conductivity values - a challenge for experimentalists?, NFSI \& ICBEM (2011) 39-43.

[9] L. Clerc, Directional differences of impulse spread in trabecular muscle from mammalian heart, J. of Physiol. 255 (1976) 335-346.

[10] D. E. Roberts, A. M. Scher, Effects of tissue anisotropy on extracellular potential fields in canine myocardium in situ, Circ. Res. 50 (1982) 342351.

[11] D. E. Roberts, L. T. Hersh, A. M. Scher, Influence of cardiac fiber orientation on wavefront voltage, conduction velocity and tissue resistivity in the dog, Circ. Res. 44 (1979) 701-712.

[12] B. J. Roth, Electrical conductivity values used with the bidomain model of cardiac tissue, IEEE Trans. Biomed. Eng. 44 (1997) 326-328.

[13] P. R. Johnston, D. Kilpatrick, C. Y. Li, The importance of anisotropy in modelling ST segment shift in subendocardial ischaemia, IEEE Trans. Biomed. Eng. 48 (2001) 1366-1376.

[14] P. R. Johnston, D. Kilpatrick, The effect of conductivity values on ST segment shift in subendocardial ischaemia, IEEE Trans. Biomed. Eng. 50 (2003) 150-158.

[15] M. C. MacLachlan, J. Sundnes, G. T. Lines, Simulation of st segment changes during subendocardial ischemia using a realistic 3-d cardiac geometry, IEEE Trans. Biomed. Eng. 52 (2005) 799-807. 
[16] B. M. Johnston, P. R. Johnston, D. Kilpatrick, A new approach to the determinination of cardiac potential distributions: Application to the analysis of electrode configurations, Math. Biosci. 202 (2006) 288-309.

[17] B. M. Johnston, P. R. Johnston, Possible four-electrode configurations for measuring cardiac tissue fibre rotation, IEEE Trans. Biomed. Eng. 54 (2007) 547-550.

[18] B. M. Johnston, P. R. Johnston, D. Kilpatrick, Analysis of electrode configurations for measuring cardiac tissue conductivities and fibre rotation, Ann. Biomed. Eng. 34 (2006) 986-996.

[19] P. C. Franzone, L. Guerri, B. Taccardi, Spread of excitation in a myocardial volume: Simulation studies in a model of anisotropic ventricular muscle activated by point stimulation, J. Cardiovasc. Electrophysiol. 4 (1993) 144-160.

[20] K. R. Foster, H. P. Schwan, Dielectic properties of tissue and biological materials: A critical review, Crit. Rev. Biomed. Eng. 17 (1989) 25-104.

[21] A. Kuntsevich, F. Kappel, SolvOpt: The solver for Local Nonlinear Optimisation Problems, version 1.1 in C, Institute for Mathematics: Karl-Franzens University of Graz, 1997.

[22] N. Z. Shor, Minimization methods for Non-Differentiable Functions, volume 3 of Springer Series in Computational Mathematics, SpringerVerlag, 1985. 
Table 1: Cardiac conductivity values (in $\mathrm{mS} / \mathrm{cm}$ ) and space constants (in $\mathrm{mm}$ ) from the studies indicated.

\begin{tabular}{ccc}
\hline & MacLachlan et al. [15] & Hooks et al. [7] \\
\hline$g_{e l}$ & 2.0 & 2.63 \\
$g_{e t}$ & 1.65 & 2.45 \\
$g_{e n}$ & 1.3514 & 1.087 \\
$g_{i l}$ & 3.0 & 2.63 \\
$g_{i t}$ & 1.0 & 0.263 \\
$g_{i n}$ & 0.35125 & 0.08 \\
\hline$\lambda_{l}$ & 0.74 & 0.77 \\
$\lambda_{t}$ & 0.53 & 0.33 \\
$\lambda_{n}$ & 0.36 & 0.18
\end{tabular}

Table 2: First pass average percentage relative errors \pm 1 standard deviation, for various noise levels, when retrieving the cardiac conductivities and fibre rotation angle for the MacLachlan et al. [15] dataset. The top of the table gives the first pass errors using a 0.5 $\mathrm{mm}$ spacing in the normal direction in the 'closely-spaced' electrode set (Figure 3(a)) and the bottom of the table is for a $0.25 \mathrm{~mm}$ normal spacing in the same electrode set.

\begin{tabular}{c|ccccccc}
\hline Noise & \multicolumn{7}{|c}{$0.5 \mathrm{~mm}$ normal direction spacing } \\
& $g_{e l}$ & $g_{\text {et }}$ & $g_{\text {en }}$ & $g_{i l}$ & $g_{i t}$ & $g_{\text {in }}$ & $\alpha$ \\
\hline $1 \%$ & $0.2 \pm 0.2$ & $0.4 \pm 0.5$ & $0.1 \pm 0.2$ & $2.0 \pm 4.7$ & $5.7 \pm 5.1$ & $2.1 \pm 12.0$ & $1.9 \pm 4.8$ \\
$2 \%$ & $0.5 \pm 0.8$ & $0.1 \pm 1.3$ & $0.1 \pm 0.6$ & $10.1 \pm 11.1$ & $19.1 \pm 19.4$ & $18.2 \pm 19.2$ & $5.4 \pm 12.8$ \\
$5 \%$ & $0.3 \pm 1.1$ & $0.3 \pm 1.9$ & $0.3 \pm 1.3$ & $20.7 \pm 24.7$ & $27.9 \pm 56.2$ & $19.3 \pm 16.9$ & $9.7 \pm 21.7$ \\
\hline Noise & \multicolumn{7}{|c}{$0.25 \mathrm{~mm}$ normal direction spacing } \\
& $g_{\text {el }}$ & $g_{\text {et }}$ & $g_{\text {en }}$ & $g_{i l}$ & $g_{i t}$ & $g_{\text {in }}$ & $\alpha$ \\
\hline $1 \%$ & $0.0 \pm 0.4$ & $0.0 \pm 0.3$ & $0.1 \pm 0.4$ & $2.7 \pm 5.3$ & $2.1 \pm 3.7$ & $17.5 \pm 36.8$ & $3.2 \pm 3.4$ \\
$2 \%$ & $0.3 \pm 0.5$ & $0.3 \pm 0.5$ & $0.4 \pm 0.5$ & $3.6 \pm 8.7$ & $3.0 \pm 13.8$ & $12.7 \pm 24.3$ & $4.4 \pm 5.9$ \\
$5 \%$ & $0.7 \pm 1.4$ & $0.5 \pm 1.5$ & $0.2 \pm 1.6$ & $28.8 \pm 40.4$ & $72.3 \pm 154$ & $207 \pm 224$ & $0.9 \pm 16.8$
\end{tabular}


Table 3: Second pass average percentage relative errors \pm 1 standard deviation, for various noise levels, when retrieving the cardiac conductivities and fibre rotation angle values for the indicated datasets, using either the 37 or 73 'widely-spaced' electrode sets (Figure $3(\mathrm{~b})$ and $(\mathrm{c}))$.

\begin{tabular}{c|cccc|cccc}
\hline Noise & \multicolumn{3}{|c|}{ MacLachlan et al. [15] } & \multicolumn{4}{c}{ MacLachlan et al. [15] } \\
& $g_{i l}$ & $g_{i t}$ electrodes & $g_{\text {in }}$ & $\alpha$ & $g_{i l}$ & $g_{i t}$ & $g_{i n}$ & $\alpha$ \\
\hline $1 \%$ & $1.2 \pm 1.1$ & $3.8 \pm 2.3$ & $0.6 \pm 1.3$ & $1.7 \pm 1.3$ & $1.3 \pm 1.2$ & $1.9 \pm 2.5$ & $0.3 \pm 1.7$ & $0.6 \pm 2.4$ \\
$2 \%$ & $3.5 \pm 2.0$ & $0.3 \pm 3.1$ & $0.9 \pm 3.8$ & $1.2 \pm 2.7$ & $2.7 \pm 2.4$ & $1.8 \pm 4.4$ & $1.4 \pm 4.2$ & $2.3 \pm 4.3$ \\
$5 \%$ & $1.1 \pm 5.5$ & $2.9 \pm 9.1$ & $6.9 \pm 7.5$ & $1.8 \pm 6.5$ & $2.4 \pm 4.8$ & $6.1 \pm 6.1$ & $11.0 \pm 11.6$ & $3.3 \pm 8.7$ \\
\hline Noise & \multicolumn{4}{|c|}{ Hooks et al. [7] } & \multicolumn{4}{c}{ Hooks et al. [7] } \\
& \multicolumn{4}{c}{37 electrodes } & & \multicolumn{4}{c}{73 electrodes } \\
& $g_{i l}$ & $g_{i t}$ & $g_{i n}$ & $\alpha$ & $g_{i l}$ & $g_{i t}$ & $g_{i n}$ & $\alpha$ \\
\hline $1 \%$ & $0.2 \pm 0.7$ & $1.4 \pm 2.7$ & $3.8 \pm 3.8$ & $0.4 \pm 0.6$ & $0.2 \pm 0.8$ & $0.3 \pm 2.1$ & $3.2 \pm 2.3$ & $0.2 \pm 0.9$ \\
$2 \%$ & $0.9 \pm 2.3$ & $10.2 \pm 5.9$ & $3.4 \pm 4.3$ & $2.0 \pm 1.6$ & $1.8 \pm 2.0$ & $0.4 \pm 5.8$ & $3.1 \pm 4.1$ & $0.7 \pm 1.9$ \\
$5 \%$ & $6.5 \pm 6.1$ & $7.4 \pm 13.6$ & $15.6 \pm 17.5$ & $0.3 \pm 5.6$ & $2.6 \pm 3.8$ & $6.5 \pm 19.4$ & $10.6 \pm 12.2$ & $0.1 \pm 5.7$ \\
\hline
\end{tabular}

\begin{tabular}{|c|c|}
\hline Tissue & $\overline{z=0}$ \\
\hline Blood & $z=1$ \\
\hline
\end{tabular}

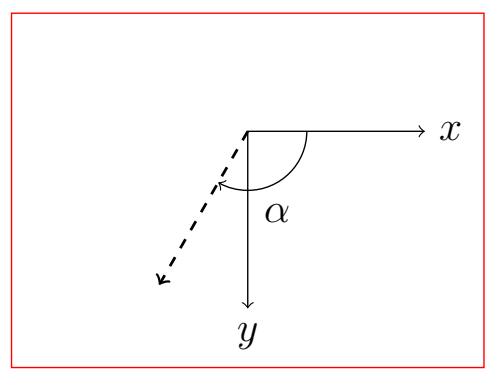

Figure 1: Two views of the tissue-blood model used in this work. In the cross-sectional view in the left panel, the positive $z$ axis is pointing downward, with the tissue lying between $z=0$ and $z=1$ and the blood at $z>1$. In the right panel is a plan view, which looks from the negative $z$-direction, the dashed arrow represents the endocardial $(z=1)$ fibre direction, when the epicardial $(z=0)$ fibre direction is along the $x$-axis and the fibre rotation angle is $\alpha$. 


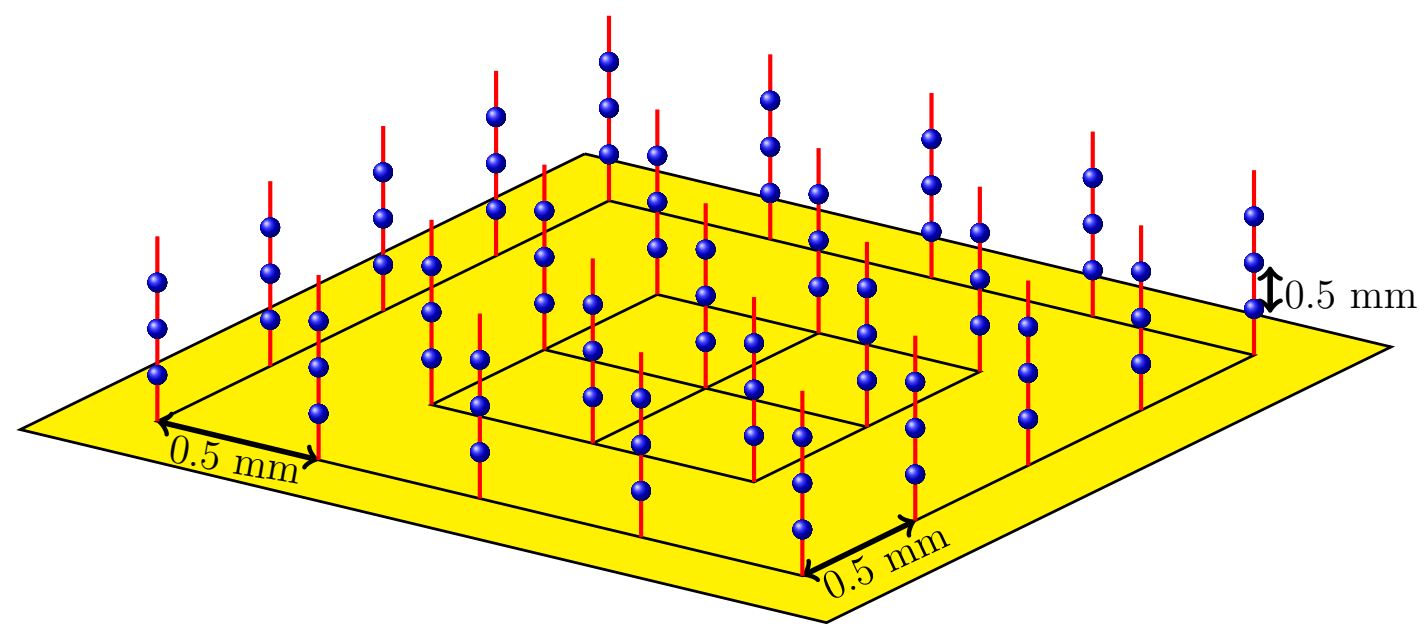

Figure 2: The full three-layer multi-electrode array proposed for retrieving six conductivity values. A different subset of the electrodes is used in each of the passes in the inversion (see Figure 3). 


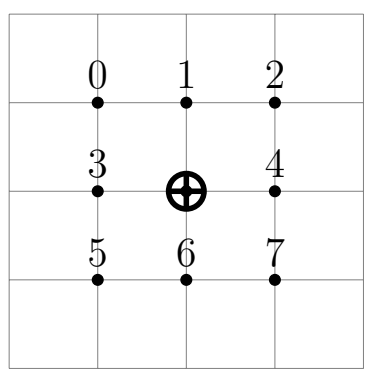

$z=0.75 \mathrm{~mm}$

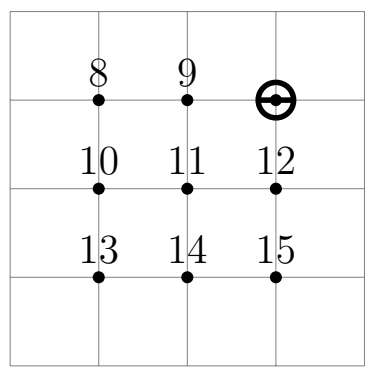

$z=1.25 \mathrm{~mm}$

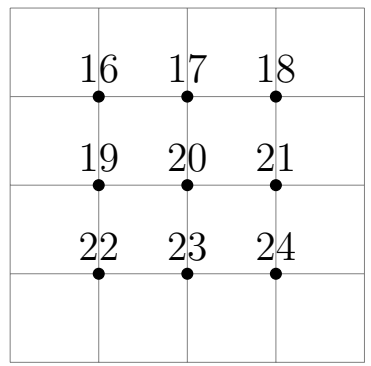

$z=1.75 \mathrm{~mm}$

(a) 'Closely-spaced' set
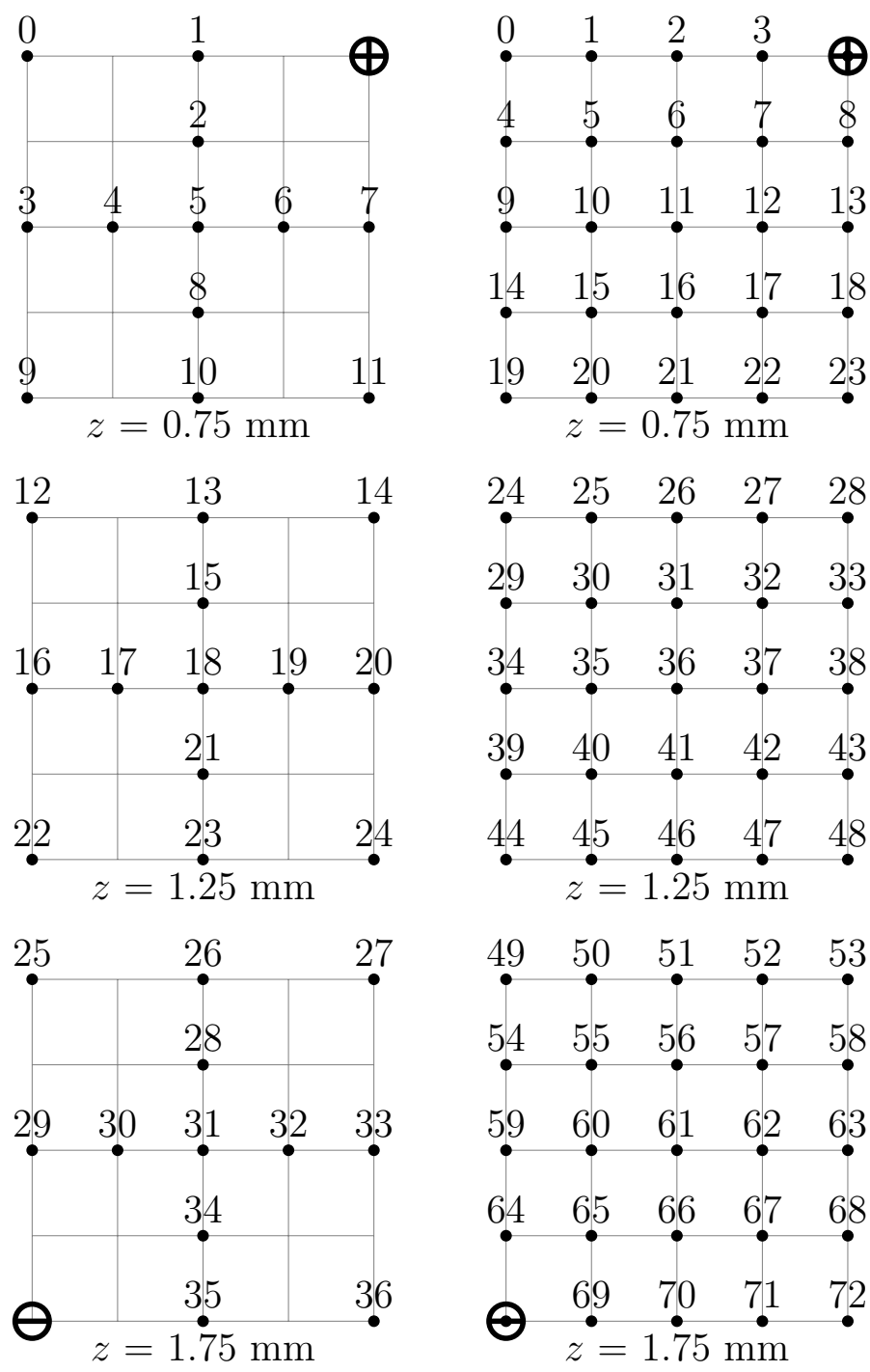

๑ $\stackrel{69}{\bullet} \underset{z=1.75}{\bullet} \quad 71 \quad 72$

$\begin{array}{lll}\text { (b) 'Widely-spaced' set } 1 & \text { (c) 'Widely-spaced' set } 2\end{array}$

Figure 3: Plan view of the three layers of the multi-electrode array, shown in Figure 2, that are used in (a) the first pass and (b) and (c) in alternative electrode sets in the second pass of the protocol to retrieve the six conductivities. The spacing between the marked squares is $0.5 \mathrm{~mm}$ in each case. Source and sink electrodes are indicated by + and - respectively. 


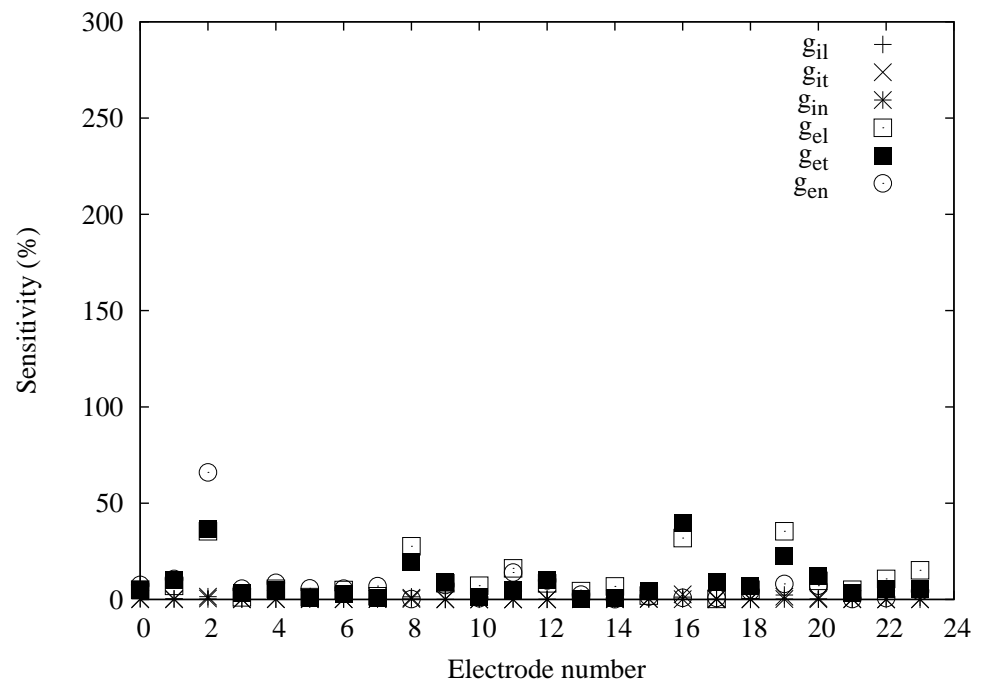

(a) 'Closely-spaced' electrode set

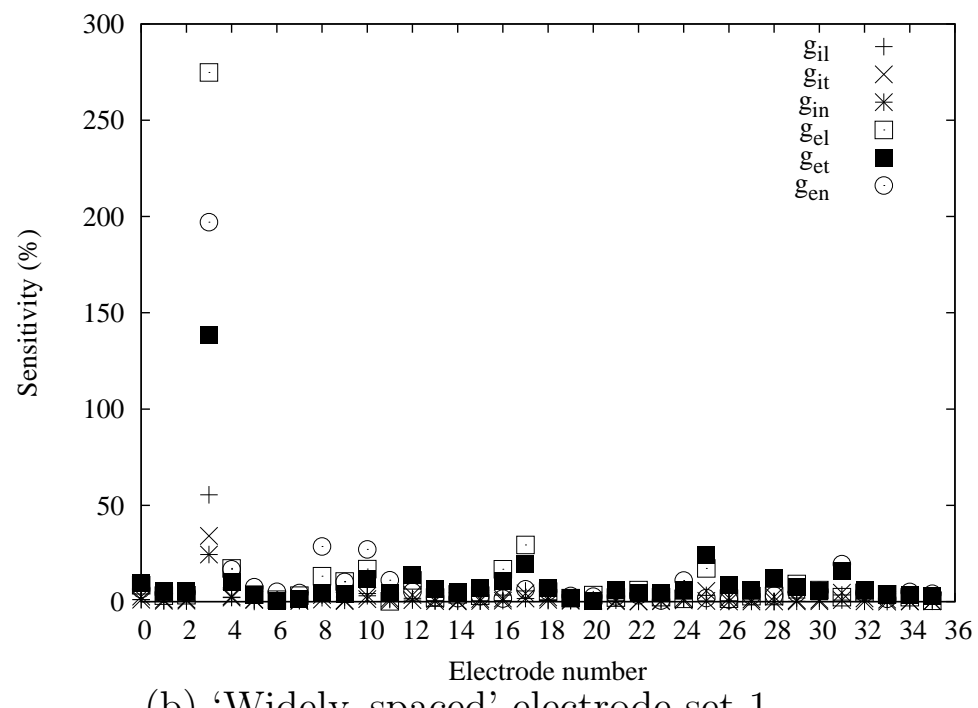

(b) 'Widely-spaced' electrode set 1

Figure 4: Sensitivity (Percentage relative error in $\phi_{e}$ ), when $10 \%$ error is added to the conductivity values, for (a) the 'closely-spaced' and (b) 'widely-spaced' electrode set 1 of Figure 3, for the MacLachlan et al. [15] conductivities. 


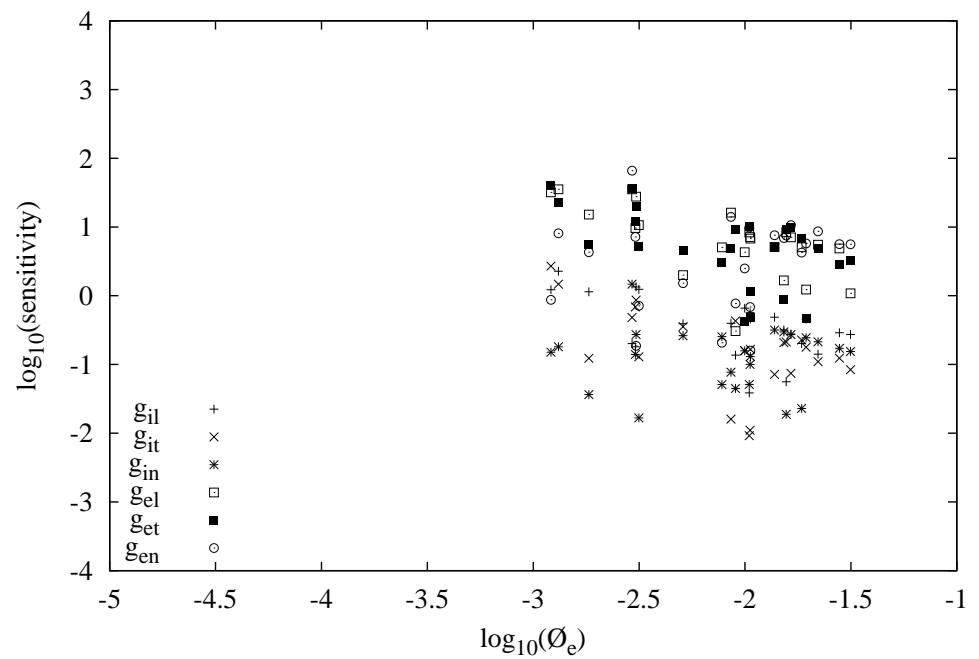

(a) 'Closely-spaced' electrode set

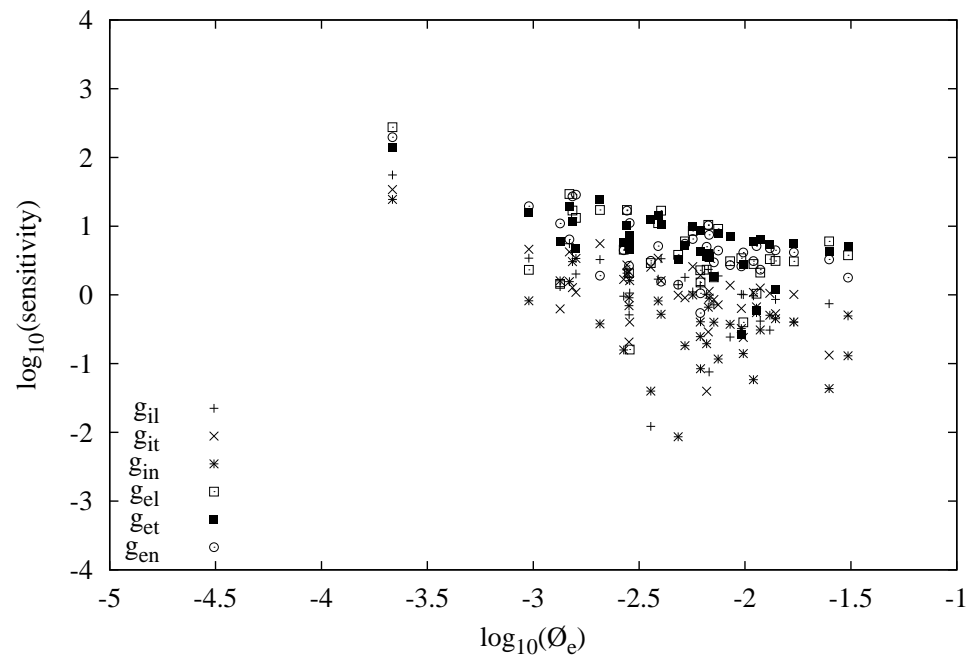

(b) 'Widely-spaced' electrode set 1

Figure 5: Sensitivity plotted against $\phi_{e}$ (on a log scale), when $10 \%$ error is added to the conductivity values, for (a) the 'closely-spaced' and (b) 'widely-spaced' electrode set 1 of Figure 3, for the MacLachlan et al. [15] conductivities. 


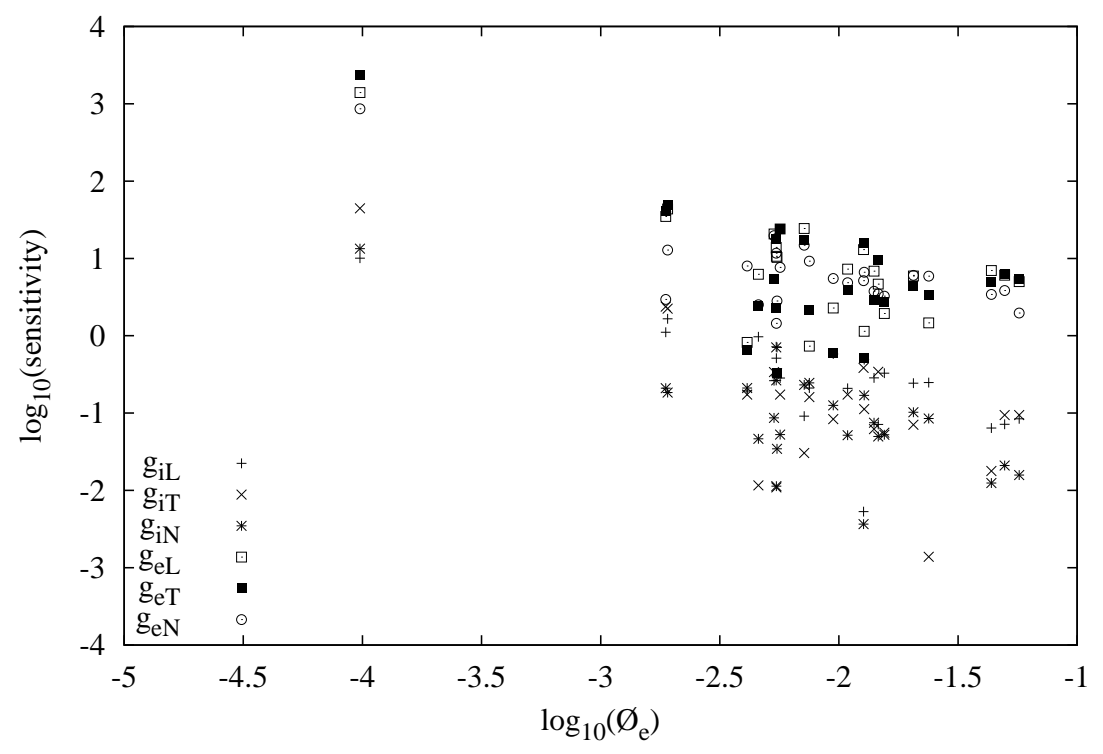

Figure 6: Sensitivity plotted against $\phi_{e}$, when $10 \%$ error is added to the conductivity values, for the MacLachlan et al. [15] conductivities. The 'closely-spaced' electrode array is the same as that used in Figure 3(a), except that the electrode spacing in the normal direction is now $0.25 \mathrm{~mm}$. 


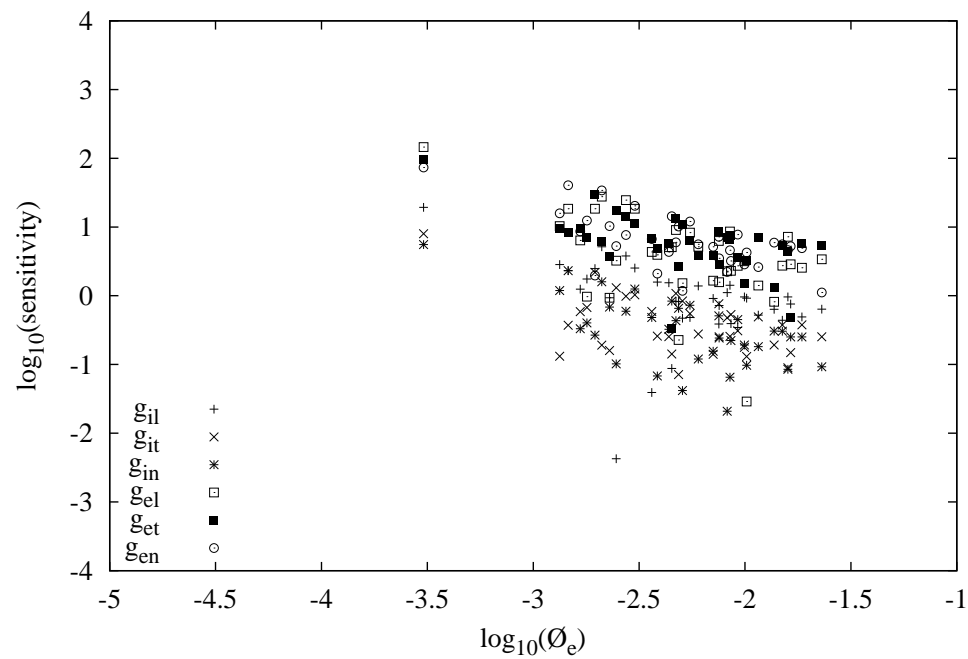

(a) 'Widely-spaced' electrode set 1

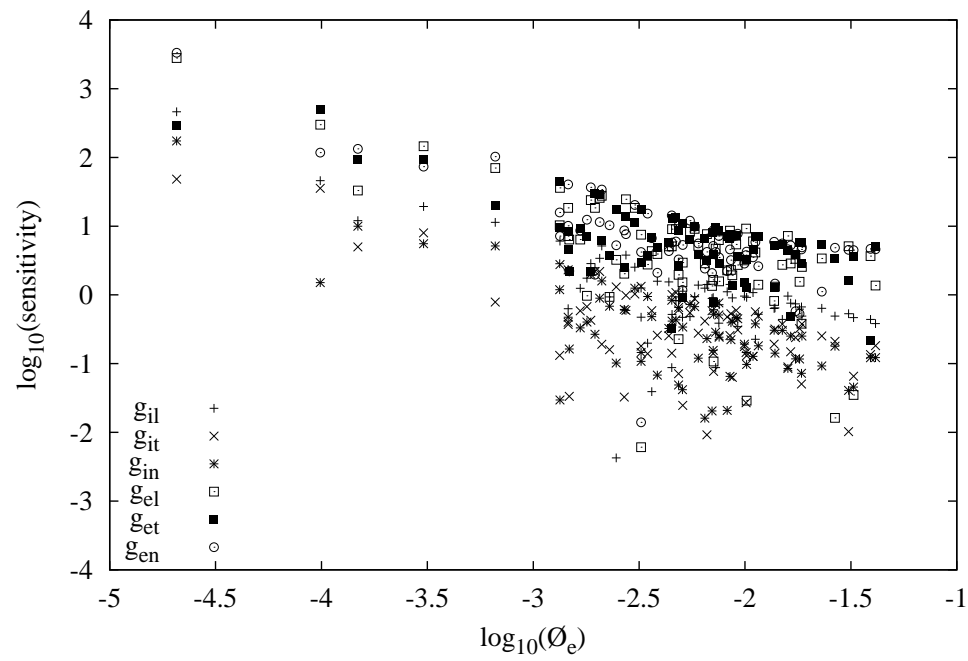

(b) 'Widely-spaced' electrode set 2

Figure 7: Sensitivity plotted against $\phi_{e}$, when $10 \%$ error is added to the conductivity values, for the Hooks et al. [7] conductivities. The 37 electrode array ('widely-spaced' electrode set 1) is shown in Figure 3(b) and the 73 electrode array ('widely-spaced' electrode set 2) is shown in Figure 3(c). 